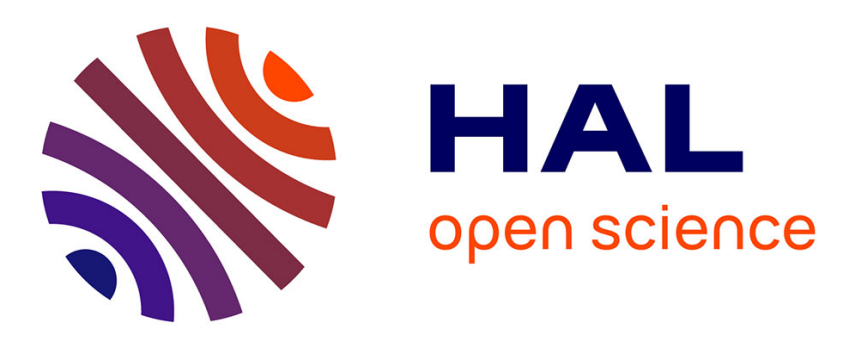

\title{
SysML Extension by AADL Specification for WSN Modeling
}

Adil Hilmani, Youssef Lasfar, Abderrahim Maizate, Abdelouahed Zakari

\section{To cite this version:}

Adil Hilmani, Youssef Lasfar, Abderrahim Maizate, Abdelouahed Zakari. SysML Extension by AADL Specification for WSN Modeling. Journal of Advanced Research in Dynamical and Control Systems, 2020, 10.5373/JARDCS/V12SP3/20201262 . hal-03235630

\section{HAL Id: hal-03235630 \\ https://hal.univ-lorraine.fr/hal-03235630}

Submitted on 25 May 2021

HAL is a multi-disciplinary open access archive for the deposit and dissemination of scientific research documents, whether they are published or not. The documents may come from teaching and research institutions in France or abroad, or from public or private research centers.
L'archive ouverte pluridisciplinaire HAL, est destinée au dépôt et à la diffusion de documents scientifiques de niveau recherche, publiés ou non, émanant des établissements d'enseignement et de recherche français ou étrangers, des laboratoires publics ou privés. 


\title{
SysML Extension by AADL Specification for WSN Modeling
}

\author{
Adil Hilmani ${ }^{1}$, Youssef Lasfar ${ }^{1}$, Abderrahim Maizate ${ }^{1}$ and Abdelouahed Zakari ${ }^{2}$ \\ ${ }^{1}$ RITM-ESTC, CED-ENSEM, Univ. Hassan II, Casablanca, Morocco. \\ \{adilhilmani, lassfaryoussef\}@gmail.com, maizate@hotmail.com \\ ${ }^{2}$ LGIPM, University of Lorraine, Metz, France \\ abdelouahed.zakari@univ-lorraine.fr
}

\begin{abstract}
In the area of software engineering and system architecture, the design and implementation process has taken a general methodological approach, encompassing all the activities necessary to design, evolve and verify a system providing an economical and efficient solution to needs of a client while satisfying all the stakeholders. For a long time, system engineers have used modeling techniques, the best known of which are AADL (Analysis and Architecture Design Language) and SysML (System Modeling Language). AADL was born as a language specific to the field focused on avionics and later was revised to represent and support a more general category of embedded systems in real time. SysML is an extension of the Unified Modeling Language (UML) to support engineering and modeling of the system. Based on these two languages and these two tools, several approaches have been created and developed to meet the needs and requirements of the system and also to overcome the problems and limitations of these two languages. In this paper, we will study and examine the two AADL and SysML modeling languages in a thorough way in order to extend the SysML language by the AADL language specifications for the modeling and design of sensor networks without (WSN).
\end{abstract}

Keywords: Modeling Process, WSN, Performance evaluation, SysML, AADL.

\section{Introduction}

In recent years, wireless sensor networks (WSNs) have performed enormously in various fields including medicine, military and environmental domains $[1,2,3]$. This sensor network is an ad hoc network made up of different sensor nodes which are electronic components whose role is to capture and measure specific parameters in a monitoring space called a capture field.

These sensor nodes are small nodes that use limited energy resources and low communication and processing power to collect information in a given geographic area and transfer them to the well node (called Sink). These limits are very important in the study, analysis and deployment of a network of wireless sensors to ensure network longevity and also to guarantee better performance and reliability in receiving data collected in a practical way and effective [4]. These sensor networks have another problem that must 
be taken into consideration and which is to model the behavioral and non-behavioral part of the latter in order to carry out the design and the deployment using the most modeling languages used to implement their functioning and behavior.

Model-based engineering (MBE) has emerged as a key set of technologies for designing complex systems. This technology has seen in recent times a tremendous progress in the realm of development and modeling of real-time systems among them wireless sensor networks (WSN). This modeling of systems is generally relying on the use of one of the two most distinguished and most used languages currently: AADL and SysML.

AADL [5] defines several categories of components, divided into three main families of components:

- The software components describe the application elements of the architecture.

- The hardware components define the elements of the execution platform.

- The hybrid components, described through the System component, are used to structure the application architecture (in blocks).

In addition, AADL supports the prediction and early analysis of critical system qualities, such as performance, planning and reliability.

SysML [6,7] is a modeling language with a graphic syntax developed and standardized by Object Management Group (OMG). It has been designed to, inter alia, capture software interactions with physical entities, and is widely used in systems engineering. Compared to UML 2, SysML adds support for systems engineering (for example, by requirements engineering and quantitative analysis of the physical aspects of the system), while removing many UML software centric constructs.

The main objective of this paper is to propose a profile-specific modeling for wireless sensor networks (WSN) based on the use of a new design technique which is based on an extension of the SysML language by adding the critical specifications of the AADL language in order to carry out and implement the modeling of wireless sensor networks.

The rest of the document is organized as follows. Section. 2 provides an overview of WSN modeling methods. In Section 3, we will explain the requirements of the SysML profiles for the modeling of WSN. Then, in Section 4 we will give a new contribution based on a proposed SysML profile. Finally, we conclude with a conclusion and a perspective in Section 5.

\section{WSN Modeling Languages}

In this section, we will study and examine the different modeling languages that are the most distinguished and used today in the design and implementation of complex and real-time systems. Among these languages are UML, MARTE, BIP, SysML, AADL. 


\subsection{Current Modeling Languages}

UML [8] (Unified Modeling Language) is a unified modeling language and Object Oriented by the OMG which is based on the use of graphs and diagrams to visualize, specify, build and document systems covering different areas of application between them the software systems, based on all standardized and object-oriented methods. UML offers thirteen diagrams that are used for the description and design of a system. These diagrams are divided into two categories of diagrams which are:

- Structural diagrams: These diagrams play a crucial role in the description of the body part of the system based on the identification of objects with their behaviors and their operations, not forgetting the operations and methods attributed to them.

- Behavior diagrams: These diagrams describe the dynamic part of a system that is based on the output of the results to the user once the system interacts with external events.

MARTE $[9,10]$ (Modeling and Analysis of Real Time and Embedded Systems) is designed to take over from its predecessor called profile for Scheduling, Performance and Time Specification (SPT), it is a recent OMG UML profile consisting of a platform and a which describes the real-time parameters and aspects of systems in UML models. This language also relies on the modeling of systems. Considering the static part, defining the components, functions and methods, and the dynamic part describing the interaction of the system with its entourage and its environment, producing real-time results. The main objective of MARTE is to create and propose a common and unique modeling language to describe and specify at the same time the software and hardware aspects of embedded systems in real time. This language consists of three major models:

- Application model: Defines aspects and functionalities of the system.

- The Resource Model: describes the implementation and execution infrastructure of the behavioral part of the system based on non-functional properties and parameters

- Allocation model: describes the interaction between applications and resources.

BIP [11] (Behavior, Interaction, Priority) is a language with a theoretical basis for the construction and analysis of embedded systems in real time from heterogeneous components. This language allows the implementation and behavioral description of real-time embedded architectures. The BIP models are obtained by the association and integration of three modeling parts:

- The lower part defines the behavioral aspect of a component or object as a set of transitions (that is, an extended state machine with data);

- The intermediate part describes the connection with the behavior part using the connectors which provide a description of the interaction between these two parts.

- The upper part consists of a group of properties and priority features used to describe the laws and rules of operation and planning of interactions. 
Modeling using the BIP language is based on the use of connectors and priorities to develop and build a hierarchical architecture of composite components from atomic components. This modeling has created an open and clear vision that has made it possible to design the behavior and operation of components and the overall structure of a system.

\subsection{Presentation of The SysML Language}

SysML [12-14] is a modeling language developed by OMG, INCOSE and AP233 and is oriented to the field of system engineering which is based on the graph to specify, analyse, describe, verify and validate the feasibility of a system before its realization. SysML has been designed to use and rely on an open source license to develop its contribution and use. SysML is defined as an extension of a subset of UML using the profile mechanism defined by UML.

The SysML language is a way to form and consolidate in a single, common model all feature components, properties, constraints, and parameters to model and design the entire system. To carry out this modeling, the SysML language is based on diagrams that make it easier to approach complex systems and also to implement their operations and their realizations.

With modeling using the SysML language, a system can be modeled and also can be designed in several manners and in different ways in order to formalize the overall behavior of the system. This freedom of modeling can create problems when implementing the realization of the operation of this system. This language does not allow the realization and the execution of the behavior of the system which limits its performance.

\subsection{Presentation of The AADL Language}

AADL (Architecture Analysis and Design Language) $[15,16]$ is an SAE international standard established in 2004 that produces a new project to analyse system designs prior to development and that supports the system architecture description for embedded systems.

The objective of the AADL language is to perform an efficient and precise modeling that includes all functional and non-functional parts of the system without forgetting all these properties and these functions such as complexity, schedulability, message transmission, hardware description (processor, memory, bus, etc.). To carry out this modeling, each component of the system is described in AADL according to two aspects. The first, type, corresponds to the functional interface of the component. The second, the implementation, describes the content of the component (subcomponents, time properties, connections, etc.). Each component belongs to one of the 10 categories predefined in AADL, classified into 3 families: hardware (Execution Platform), software (Application Software) and hybrid (Composite). 
To model an architecture, the AADL components must be declared as subcomponents (instances) of other AADL components. At the highest level, a system component contains all instances of other components. Most AADL components can contain subcomponents that allow a hierarchical description of the system.

\section{SysML Profiles Requirements for WSN Modeling}

This paper proposes a new process of modeling wireless sensor networks that is based on the combination of two languages SYSML and AADL precisely on the notion of profile in UML/SYSM, this notion which allows specializing a part of the AADL language by SYSML in order to produce a profile that will provide a complete modeling support for WSN designers.

\subsection{The SysML-AADL Profil}

The initial experiments with the SYSML and AADL modeling languages have revealed difficulties in handling these tools. Certainly, the syntax of these languages is very wealth, but the semantics and methodology to be adopted remain dependent on the application field and the context of use. Hence the idea of specializing and customizing a profile dedicated to sensor networks based on the complementarity of these two languages and the possibility offered by extensions with SYSML profile (which is also a UML profile). The ultimate goal is to create a specific profile for wireless sensor networks by integrating new features based on feedback from these two languages.

\subsection{SysML-AADL Profile requirements for WSN}

Generally modeling is a very important operation when it is poorly mastered it can engender a model that does not exactly represent the system and which, in some cases, can cause defects in the behavior or execution of the system which in turn leads to a renunciation of the solution.

In our case, we proposed a new profile of modeling and analyzing SYSML-AADL that will be served as a support for the designers and developers of the WSN. Indeed, a WSN is based on four components:

- Nodes containing sensors.

- A wireless interconnection networks.

- A base station for grouping information and acting as an interface between the nodes and the user.

- Processing resources at the base station level to manage data correlation.

The SYSML-AADL profile must consider all the requirements related to these components (lifetime, scheduling, communication, etc.) in order to produce a complete solution which allows building a complete support for the modeling of the WSN. In this part, we will describe all these requirements which are divided into four categories:

- Requirements related to the structure of WSN. 
- Requirements related to the behavior of WSN.

- Requirements related to energy constraints.

- Specific requirements.

\subsubsection{Requirements related to the structure of WSN}

A wireless sensor network consists of a group of small wireless node networks; each node group is connected to a gateway. The gateways send the collected data to a base station via a transmission network.

In order to model this structure, our profile must support the following requirements:

- REQU STRUCT-1: The modeling of the sensor nodes and their characteristics (commercial name, operating systems supported ...) as well as the internal components that ensure the communication and the data processing (ex: transducer, processor).

- REQU STRUCT-2: The modeling of communication links between two devices (between two sensor nodes for example) and their characteristics (error rate ....)

- REQU STRUCT-3: The modeling of sensors, gateways and base stations and their characteristics.

- REQU STRUCT-4: The modeling of the transmission network specification.

\subsubsection{Requirements related to the behavior of WSN}

In addition to collecting and transmitting data a wireless sensor network can also perform other tasks, such as changing behavior in the event of a failure.

- REQU BEHAVIOR-1: Modeling the process of data collection.

- REQU BEHAVIOR-2: The modeling of the maintenance process (periodic sending of messages to indicate the state of health of a sensor node).

- REQU BEHAVIOR-3: Modeling the configuration process and auto-reconfiguration

\subsubsection{Requirements related to the energy constraints}

Energy conservation is an important issue for WSN as the nodes contain a limited amount of energy, which cannot usually be renewed.

REQU ENERG-1: our profile must define the modeling entities for the specification of the energy properties of the different elements of the model.

\subsubsection{Requirements related to the specific constraints}

The scope of use of the WSN is very wide, which generates a specific constraint related either to the environment of use or to the mechanism of configuration of the network. 
However, our SYSML-AADL profile must be scalable and extensible to support any possible constraints.

\section{Contribution: Proposal of a SysML Profile}

\subsection{Why a Specific Profile definition?}

As we saw in the previous section, SysML is an ideal language to serve the systems modeling as the WSNs without forgetting the AADL. We now have to make the link between these two languages. We set ourselves the objective of demonstrating the interest of such a combination by first developing a SysML profile for AADL. As we know the ideal would be to develop a DSL (Domain Specific Language), completely dedicated to the WSNs. This objective will interest us in a second time and we chose at first to start pragmatically by defining a SYSML profile, The SysML profile will solve a number of AADL language limitations:

- It will provide a development environment and graphic support for the expression of the constraints of the WSNs.

- It will provide SysML tools that are now starting to be used in industrial settings.

- It will make it possible to check the consistency of the operators defined by their implementation in OCL constraints.

- It will make the transition between the expression of needs and the analysis in an efficient way and with a minimum of information loss (seamless).

The Expected progress of the project and the expected results:

The development of this profile is not the mean goal. It is part of a thesis aiming at the elaboration of a set (WSNs modeling and code generation as long as testing) of software tools for the modeling of the WSNs.

The need for the development of a SysML profile supporting the expression and taking into account AADL elements. The idea is to make (starting with UML) the taxonomy of the types of association. First intra-model (relations, compositions, etc. in a class diagram for example), then inter-model (as dependencies), with in this case the mono levels of meta-modeling (M1) or multi-levels (M1 / M2, for example the type conforms to).

\subsection{Mapping Component Type and Component Implementation}

In AADL, component types and component implementations describe the component, respectively, the visible interfaces and the internal structure. In our case, we will use the blocks for the modeling of the component's types and the components implementations of AADL. To differentiate between these last two, we will use two new stereotypes, "ComponentType" and "ComponentImpl", two SysML extensions.

The SysML blocks are extensions of the UML classes, they are chosen for the modeling of the components to describe the two structural and behavioral parts of the system, by adding only the block modeling, this will allow the use of other SysML 
constructs (ports and parts) for consistent modeling of AADL parts (subcomponents and ports).

AADL provides ten components of different categories. For each identifier in the AADL category, we reserve a stereotype with attributes representing the properties of the chosen category. The values associated with the AADL properties are automatically projected as values of the attributes of the stereotypes applied to the blocks. All category identifiers are generalized by "ComponentType" and "ComponentImpl". We apply two stereotypes for each block: a stereotype specifies whether it is ("ComponentType") or it is ("ComponentImpl"), and the other will specified its component categories.

In AADL, the "ComponantImpl" can refine the specification of the "ComponantType" by adding implementation details. For example, specifying the subcomponents, connections, modes, properties and the flow.

A realization relationship in the AADL enables all the values of the "ComponantType" properties to be transferred to "ComponantImpl", and gives accessibility to all aspects and specifications of the "ComponantType" flows, from the "ComponantImpl".

For our Model in UML / SysML, this is done by "AADL_Realization", each relation of realization in our model must be stereotyped by "AADL_Realization".

\subsection{Application and Evaluation of the Profile}

In this section, we conducted a case study based on wireless sensor network modeling using the profile proposed in the previous section. In this true study, we will evaluate the capability of this profile to meet the modeling requirements of a wireless sensor network system (WSN) consisting of two sensor nodes, a Head cluster node and a base station using AADL/OSATE.

To start the study, first you need to create a "ComponentType" system type that allows to gather all the different components needed to structure the architecture of the sensor network. In our case, we will create two nodes, a $\mathrm{CH}$, and a BS. For example, specifying the subcomponents, connections, modes, devices, properties and the flow. To accomplish this task, it is necessary to model the implementation part "ComponentImpl" of this "ComponentType" in order to refine and define its internal structure in terms of interactions between the different subcomponents (see Fig. 1). 


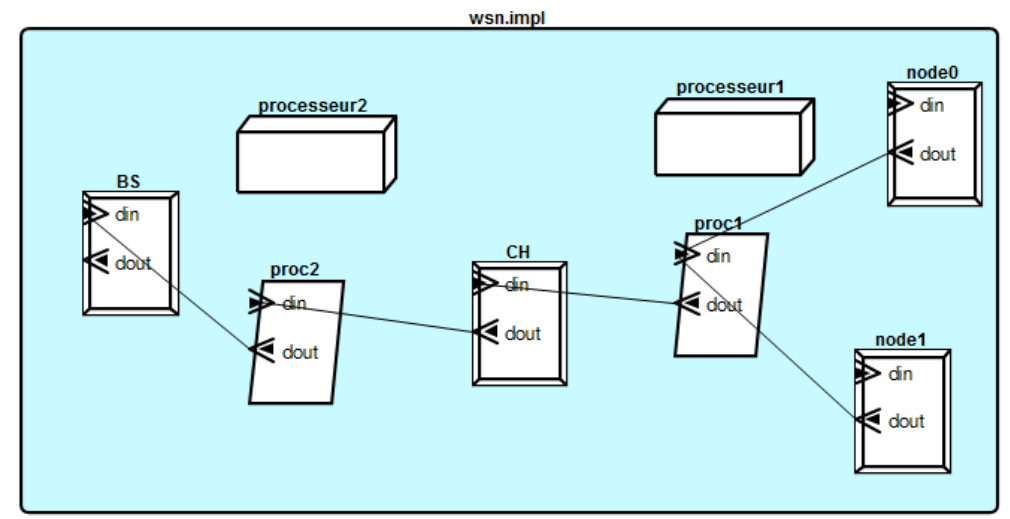

Fig. 1. Iimplementation of the proposed system WSN

After modeling the internal architecture of the system, it is important to define the functions and processes to be executed for each component of the system. To achieve this part, you need to create a new "ComponentType" type process that defines the function to perform for each component using "ComponentImpl" to refine the behavior of the component with more detail and accuracy. In our case, it is necessary to determine the tasks that will be executed by the 2 nodes, the $\mathrm{CH}$ and the base station without forgetting to define the technique of access control to the wireless communication medium which will allow to transmit several flows of traffic on a single channel in real time (using TDMA).

Once the processes to be performed by the WSN system have been completed, the tasks to be performed for each network component are obtained. As shown in the following Figures:

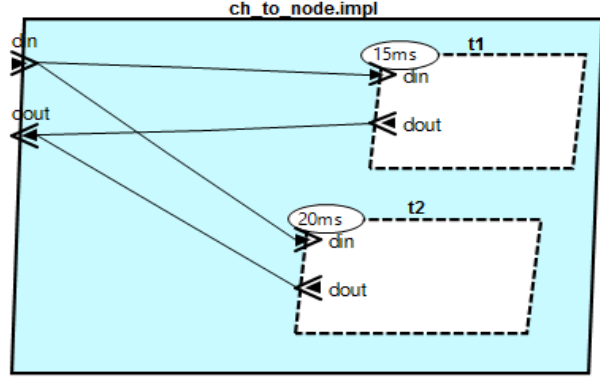

(a)

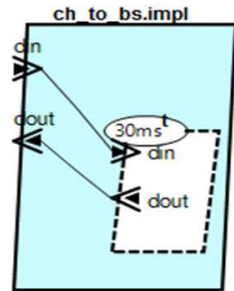

(b)

Fig. 2. a) Implementation of $\mathrm{CH}$ processes, b) implementation of BS processes

According to the study carried out, we can conclude that the developed model based on the extension of SysML using the AADL specifications has gathered all the features and details to model the WSN wireless sensor network system. As expected, many aspects of the SysML model required the use of AADL features because SysML proved to be insufficient and powerless. 


\section{$5 \quad$ Conclusion and Perspectives}

The works presented in this article are "in progress". Our study of the state of the art makes it possible to show the pluridisciplinary and applied nature of the research that can be done within a framework of the modeling of the WSNs. The concrete development of an experimental platform will demonstrate the interest of the approach described and raise other questions both practical and theoretical on the development of hardware / software-based engineering models.

\section{References}

1. Samira Kalantary, Sara Taghipour, "A survey on architectures, protocols, applications, and management in wireless sensor networks". Journal of Advanced Computer Science \& Technology 3.1 (2014): 1-11

2. Lan F.akyildiz and mehmet can vuran "wireless sensor networks". wiley publication,2010.

3. Akyildiz IF, Su W, Sankarasubramaniam Y, Cayirci E. A survey on sensor networks. IEEE Communications Magazine. 2002; 40:102-14.

4. Adil Hilmani, Abderrahim Maizate. A Study of Self-Organization protocols in Wireless sensor Network. Mediterranean Telecommunications Journal, Vol. 7, N² 2, July 2017.

5. R. Behjati, S. Nejati, L. Briand, B. Selic, An AADL-Based SysML Profile for Architecture level Systems Engineering: Approach, Metamodels, and Experiments. 2011.

6. SysML, http://www.sysml.org.

7. N. Belloir, J.-M. Bruel, N. Hoang, and C. Pham, Utilisation de SysML pour la mod'elisation des r'eseaux de capteurs sans fil, Conf'erence sur les Langages et Mod`eles `a Objets (LMO), Montr'eal, Canada, 03/03/2008-07/03/2008, pages 171 186, http://www.cepadues.com/, mars 2008 .

8. G Booch, I Jacobson, J Rumbaugh. The unified modeling language, Unix Review, 1996 ccs.neu.edu

9. C André - Université Nice Sophia Antipolis, Le temps dans le profil UML MARTE, 2007 wwwi3s.unice.fr, UNSA-CNRS

10. OMG: UML profile for Modeling and Analysis of Real-Time and Embedded systems (MARTE), beta 1, August 2007, document ptc/07-08-04.

11. MY Chkouri, Modélisation des systèmes temps-réel embarqués en utilisant AADL pour la génération automatique d'applications formellement vérifiées, 2010 - hal.archives-ouvertes.fr

12. http://www.uml-sysml.org/sysml

13. T Weilkiens, Systems engineering with SysML/UML: modeling, analysis, design, 2011.

14. PH Feiler, DP Gluch, JJ Hudak, The architecture analysis \& design language (AADL): An introduction, 2006 - dtic.mil

15. PH Feiler, BA Lewis, S Vestal, The SAE Architecture Analysis \& Design Language (AADL) a standard for engineering performance critical systems, Computer Aided Control System Design, 2006 IEEE International Conference on Control Applications, 2006 IEEE International Symposium on Intelligent Control, 2006 IEEE

16. Hugues J., Singhoff F., « Développement de systèmes à l'aide d'AADL - Ocarina/Cheddar », Tutoriel présenté à l'école d'été temps réel, Paris, Septembre 2009. 